\title{
Factores implicados en la publicación científica: una revisión crítica
}

\author{
Factors involved in a scientific publication: a critical review \\ Francisco Ganga $^{1} \quad$ Juan Castillo $^{2} \quad$ Liliana Pedraja-Rejas ${ }^{3 *}$ \\ Recibido 28 de abril de 2016, aceptado 10 de agosto de 2016 \\ Received: April 28, $2016 \quad$ Accepted: August 10, 2016
}

\begin{abstract}
RESUMEN
Un artículo apto para ser publicado precisa dar cuenta de nuevos adelantos en distintas áreas de conocimiento, debe ser fruto de una minuciosa labor de búsqueda, de consulta, de una exhaustiva revisión bibliográfica en distintas fuentes y, por supuesto, es preciso cuidar las normas gramaticales y la redacción. Gracias a la investigación los seres humanos desarrollan la ciencia y la tecnología, indagan y logran entender el pasado, conocen la evolución de su entorno y muchas veces consiguen perfilar el futuro. La labor de quienes se dedican a la investigación es constante, seria y paciente; sin embargo, su quehacer cobra mayor sentido cuando se da a conocer, y para ello es indispensable la divulgación de los resultados alcanzados. Este estudio tiene como finalidad ofrecer algunas reflexiones sobre la significación de la investigación y los elementos a considerar al momento de enviar un trabajo a una revista académica o científica para su evaluación y posterior publicación. Desde el punto de vista metodológico, el estudio se basa en la revisión de fuentes secundarias (revistas, artículos científicos y bases de datos) y también en la experiencia acumulada por los autores. Se espera contribuir tanto al diálogo académico como a las tendencias actuales de circulación del conocimiento científico, por medio de una reflexión crítica.
\end{abstract}

Palabras clave: Investigación, revistas académicas, publicación científica, universidades.

\begin{abstract}
An article considered for publication should make known new developments in different knowledge areas, it must be the result of a thorough research, a comprehensive literature review on different sources and, of course, it is important to be careful with grammatical rules and good writing. Through research human beings develop the science and technology, investigate and come to understand the past, know the evolution of their environment and often achieved shape the future. The work of those engaged in the research is constant, serious and patient. However, their task only makes more sense when it is disclosed, and for this is indispensable the dissemination of the results achieved. This study aims to offer some reflections on the significance of research and the elements to consider when sending an article to an academic or scientific journal for its evaluation and subsequent publication. From the methodological point of view, the study is based on the review of secondary sources (review of journals, scientific articles and databases) and also on the experience gained by the authors. It is expected to contribute to both the academic dialogue as current trends of scientific knowledge circulation through critical reflection.
\end{abstract}

Keywords: Research, academic journals, scientific publication, universities.

1 Departamento de Ciencias del Desarrollo. Universidad de Los Lagos. República N ${ }^{\circ}$ 517. Santiago, Chile.

E-mail: fganga@ulagos.cl

2 Departamento de Ciencia Política. Instituto de Formación Docente Continua. San Luis, Argentina. Doctorando ULSETB de Bélgica. E-mail: juancastillosl@yahoo.com.ar

3 Centro de Estudios CEUTA. Universidad de Tarapacá. 18 de Septiembre 2222. Arica, Chile.

E-mail: lpedraja@uta.cl

* Autor de correspondencia 


\section{INTRODUCCIÓN}

Publicar en una revista académica debiera representar el punto culmine de toda investigación. Ello posibilita la divulgación de los resultados de un trabajo minucioso y muchas veces largo y paciente de uno o varios investigadores. Solo cuando este quehacer sale a la luz y es puesto al servicio de la comunidad, la investigación cumple verdaderamente con su razón de ser.

Se señala lo anterior, pues nadie podría poner en tela de juicio que la labor investigativa es de extrema relevancia, dado que permite al individuo escudriñar en diferentes temas, progresar y desarrollarse. A partir de esa constante búsqueda del investigador, la sociedad ha podido responder a inquietudes y despejar preocupaciones, desarrollar la ciencia y la tecnología, ahondar y entender el pasado, conocer y evaluar el contexto, pero sobre todo proyectar el futuro a partir de la información. La investigación responde a la curiosidad y brinda la posibilidad de buscar soluciones transformándose en un poderoso acicate para la actividad intelectual innovadora-creadora. Otro aspecto, no menos trascendente, concierne al acercamiento del académico o investigador a la realidad mediante su quehacer diario.

Según Ander-Egg [1], la investigación surge de manera informal, prácticamente con el hombre mismo; desde que este se enfrentó a problemas y empezó a preguntarse el por qué, el cómo, el cuándo y el para qué, comenzó a indagar, a investigar.

Ahora bien, podría decirse que en la actualidad la investigación científica se origina de la misma forma: a partir de una inquietud cognitiva, de una situación problemática brota la necesidad de auscultar fenómenos para descubrir acerca de lo que no se tiene precisión o es desconocido.

En este orden de cosas, se espera contribuir a la reflexión y al diálogo académico y a las tendencias actuales de circulación del conocimiento, por medio de la presentación de algunos factores críticos de éxito en la publicación de artículos. Para ello, se recurre a fuentes secundarias, esencialmente revisión bibliográfica (análisis documental) y también a la experiencia sistematizada de los autores.

Tomando en consideración estos antecedentes, el trabajo intenta abordar: la preeminencia de la investigación, el valor e incidencia de la socialización de sus resultados, los criterios para llevar adelante su publicación, el impacto de la generación de redes que faciliten la comunicación y, finalmente la estructura detallada de un artículo.

\section{ANÁLISIS CRÍTICO}

\section{Importancia de la investigación: antecedentes preliminares}

En primera instancia se entregan algunos elementos que permitan determinar el alcance de su significado. En este sentido, la acepción del término investigación de acuerdo con la Real Academia Española [2] proviene del latín investigatı̌o -ōnis, que lo define como: acción y efecto de investigar.

A su vez, para investigar, se pueden destacar dos significados:

1. tr. Hacer diligencias para descubrir algo.

2. tr. Realizar actividades intelectuales y experimentales de modo sistemático con el propósito de aumentar los conocimientos sobre una determinada materia.

Desde el punto de vista filológico, Gómez de Silva [3] en su Breve Diccionario Etimológico de la Lengua Española indica que este concepto está referido a la acción de examinar sistemáticamente, observar, tratar de descubrir: del latín investigare "buscar cuidadosamente, seguir la pista, descubrir".

El empleo más ordinario de la expresión apunta a averiguar diligentemente o descubrir algo. El uso y la costumbre han llevado a emplear el vocablo siempre relacionado con actividades orientadas a la búsqueda, la indagación, la pesquisa.

En el ámbito científico, la investigación se torna un proceso reflexivo, sistemático, controlado y crítico cuya finalidad es descubrir o interpretar los hechos y los fenómenos, las relaciones y las leyes de una determinada esfera de la realidad [1]. Ello involucra generalmente una serie de etapas: formulación y definición de problemas, planteo de hipótesis, recopilación, sistematización y elaboración de datos, enunciación de las deducciones y las proposiciones generales y, finalmente, el análisis de los resultados o las conclusiones a las que se ha llegado para determinar si se confirman o no las hipótesis planteadas, y si se ajustan al marco teórico 
del que se partió. En la Figura 1, se pueden observar ocho características que sintetizan el pensamiento de Ander-Egg.

Cuando un académico se enfrenta a un proceso investigativo, le surgen una serie de interrogantes como:

- ¿Cuál es el sentido de investigar?

- ¿Cuál es la importancia de la generación del conocimiento?

- ¿En qué contexto se produce la investigación?

- ¿Cuánto se le enseña a los estudiantes universitarios acerca de la metodología de investigación?

- ¿Cuánto saben los docentes acerca de la investigación?

- ¿Cómo se conectan los nuevos saberes generados con el ejercicio de la docencia?

- ¿Qué nivel de uso tienen los conocimientos que se generan en una investigación?

- ¿Qué importancia tiene para los directivos universitarios el proceso y los resultados investigativos?

- ¿Cómo dar a conocer a la sociedad los resultados de las investigaciones realizadas?

- Entre muchas otras.
$\mathrm{Al}$ respecto, lo primero que se puede indicar es que la investigación debiera ser el eje conductor del ciclo formativo. Por su gran impacto en el progreso de los países, y por estar estrechamente ligada al avance de la ciencia, demanda de un profundo conocimiento del método científico.

El propio avance del conocimiento impone la necesidad de contar cada vez más con docentes capaces de aportar al proceso investigativo.

En las universidades regularmente se imparten principios básicos de metodología de la investigación, a pesar de ello no siempre se logra motivar a los estudiantes para continuar investigando. Aquellos que deciden aportar y avanzar en este campo deben transitar por el camino académico o luchar por incorporarse a algún centro o instituto. Esto debido a que en el contexto iberoamericano, si bien el sector privado invierte grandes sumas de dinero en investigación aplicada, usualmente son las universidades las que continúan siendo el pilar de la formación y transmisión del conocimiento.

Siguiendo esta misma línea de análisis, según datos publicados por Gutiérrez y Mayta [4],

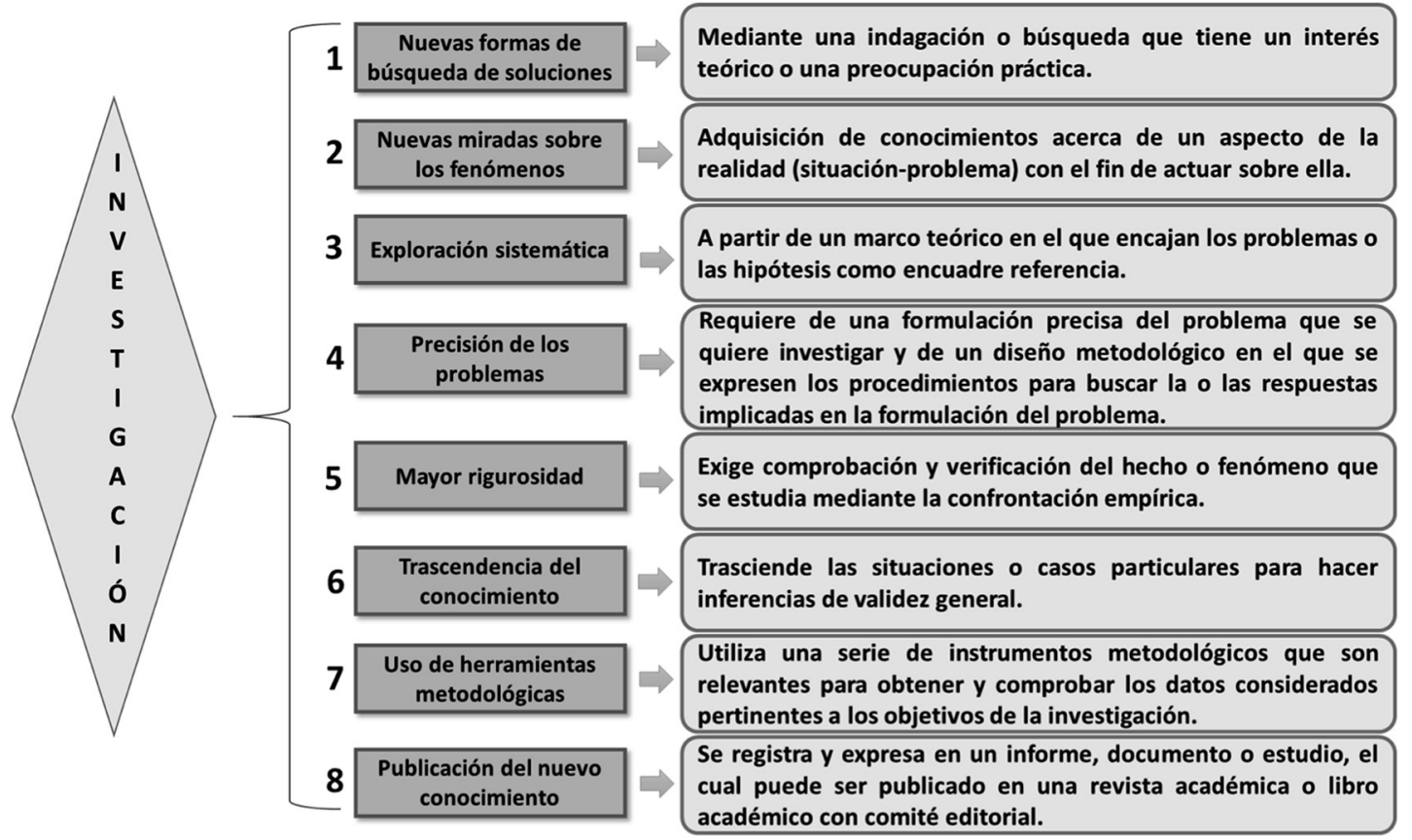

Figura 1. Ocho características distintivas de la investigación.

Fuente: Diseño propio, basado en Ander-Egg [1]. 
los estudiantes que desean profundizar sus conocimientos y aplicarlos a la investigación son una minoría en Latinoamérica. Los autores afirman, por ejemplo, que en Venezuela solo el 27\% de los alumnos, en el área de medicina, logró terminar un trabajo y presentarlo en un congreso. Sustentan además tales afirmaciones con el ejemplo de una encuesta realizada en Perú, la que arrojó que si bien los alumnos realizaron investigaciones y las compartieron en congresos, ninguno de ellos llegó a publicarlas.

En este sentido, y citando ahora a Day [5], se puede afirmar que la publicación científica es parte esencial del proceso de investigación, por lo que es factible considerar que un trabajo investigativo no está completo mientras los resultados no se hagan públicos de manera formal. El objeto principal de la investigación es el de generar nuevo conocimiento y este solo puede ser conocido y aplicarse en un campo determinado si es debidamente difundido. Bajo este criterio, las investigaciones carecen de sentido si no sirven a la sociedad, siendo necesario que se publiquen en alguna revista o libro especializado que las disemine.

Para lograr lo anterior, el proceso debe iniciarse con la idea de investigación, continuar con el desarrollo de un proyecto apropiado, luego con la ejecución y la presentación del informe final y culminar en la respectiva publicación, la que debe dar cuenta de los nuevos saberes que se han generado.

En esta misma línea, se podría aseverar también que el conocimiento se convierte día a día en la principal fuente de poder y de riqueza de las naciones [6]; esta idea no es nueva, pues ya lo exponía con mucha nitidez el filósofo, poeta, pintor y novelista libanés, Khalil Gibrán, cuando expresaba: "la verdadera riqueza de una nación no consiste en su oro ni en su plata, sino en su saber, en su sabiduría y en la rectitud de sus hijos" [7].

Por lo tanto, la generación de equipos y redes de investigación nacionales e internacionales, el apoyo y el reconocimiento a la función investigativa (alta reputación de los investigadores) y el desarrollo de acciones concretas para prestigiar estas agrupaciones, debería ser de carácter estratégico para los estados y los empresarios, dada su incidencia en el desarrollo de los países.

\section{PUBLICACIÓN DE UN ARTÍCULO DE INVESTIGACIÓN: ELEMENTOS A TENER EN CUENTA}

\section{Consideraciones generales}

Como ha quedado de manifiesto, la publicación es el testimonio final y el corolario del esfuerzo de la labor realizada, marca presencia en la comunidad científica, deja registro y difunde el quehacer del investigador ante el mundo académico. Publicar, significa además validarse y trascender, brindar acceso a los pares, a los estudiantes y a la comunidad en general sobre los resultados obtenidos, contribuye a que el conocimiento generado se transforme en algo útil para la sociedad.

Por otra parte, la publicación científica cobra relevancia dada su incidencia en la evaluación de la carrera docente y del currículum al momento de intentar avanzar en el mundo universitario o de obtener el financiamiento de un proyecto de investigación.

Es muy importante considerar que la tarea de publicar tiene sus complejidades -las que aumentan, dependiendo de la calidad de la revista- por lo que al momento de intentar publicar en una serie de problemas; los más significativos, se pueden apreciar en la Figura 2.

\section{Consideraciones específicas}

Dado que la calidad y el número de publicaciones de un académico son la mejor forma de evaluar su productividad científica, resulta beneficioso valorar detenidamente algunas cuestiones básicas: en cuál revista publicar, pertinencia de su comité y línea editorial, si utiliza el sistema de revisión de pares, si la misma está indexada a nivel nacional o internacional, cuál es su Factor de Impacto (FI), entre otros aspectos.

En este orden de cosas y tomando como basamento la experiencia en la publicación de artículos, y el trabajo de Ganga, Paredes y Pedraja [8], a continuación se presentan una serie de recomendaciones a tener en cuenta, antes de seleccionar la revista a la que se enviará el artículo.

1. Indexación: Saber si la revista está o no registrada en alguna base de datos bibliográfica de relevancia: ISI (Institute for Scientific Information, de Thomson 


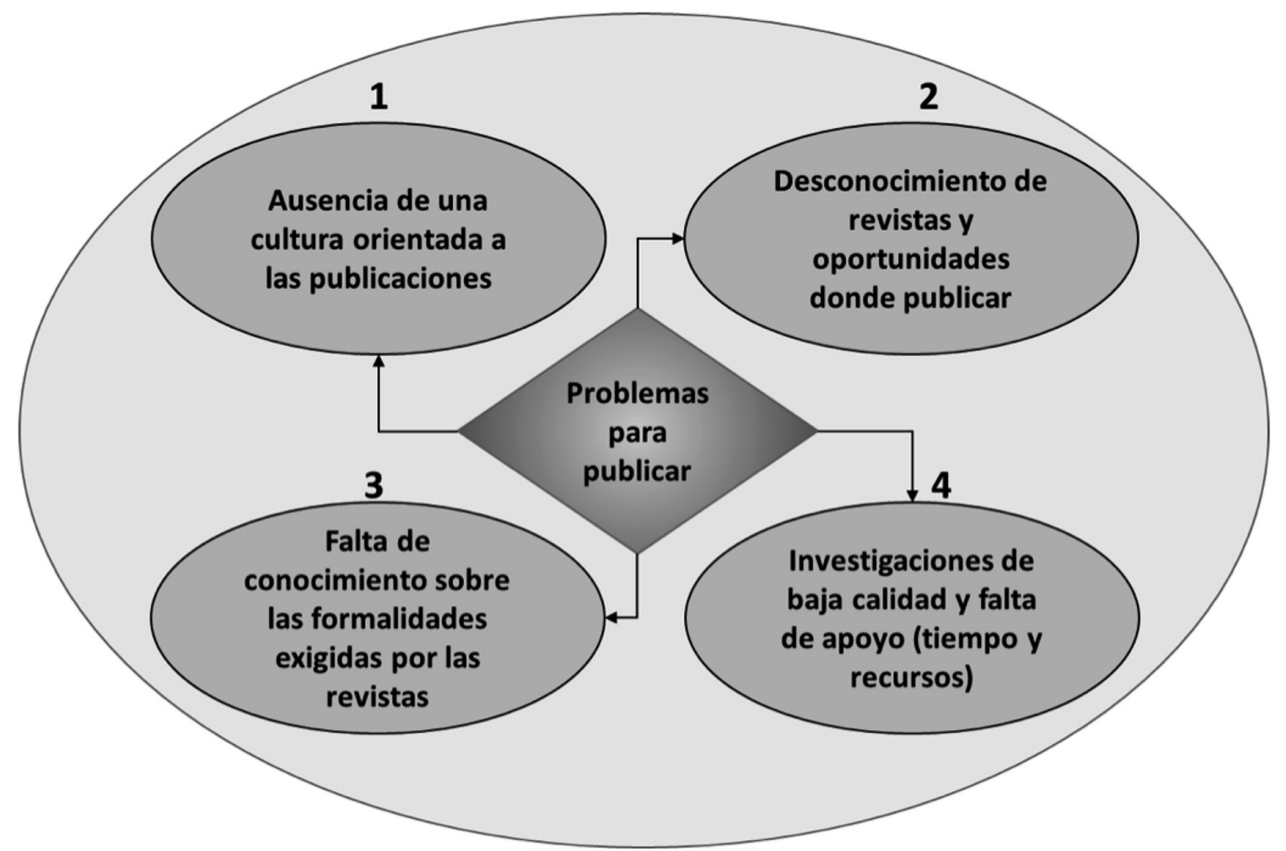

Figura 2. Problemas que se deben enfrentar a la hora de publicar.

Fuente: Diseño propio, basado en Gutiérrez y Mayta [4].

Reuters), Scopus (editada por Elsevier), Scielo (Scientific Electronic Library Online o Biblioteca Científica Electrónica en Línea), Latindex (Sistema Regional de Información en Línea para Revistas Científicas de América Latina, el Caribe, España y Portugal), Redalyc (Red de Revistas Científicas de América Latina y el Caribe, España y Portugal), entre otros.

2. Idioma: Analizar y conocer el idioma que aceptan las revistas también es muy relevante. Aunque el mayor número de revistas indexadas están en inglés, los investigadores latinoamericanos encuentran menos obstáculos para publicar en su idioma nativo; no obstante, se debe tomar en cuenta que es menor la cantidad de publicaciones especializadas de países hispanos. En todo caso, también son una muy buena alternativa, la gran cantidad de revistas que se editan en portugués (especialmente de Brasil).

3. Periodicidad: Si la revista publica una mayor cantidad de números al año, puede que existan más posibilidades de acortar los tiempos. Algunas aparecen solo semestralmente, mientras otras cada tres, dos y hasta anualmente, con un número cada mes.
4. Cantidad de artículos: Si la revista publica una mayor cantidad de artículos por número, podrían existir mayores posibilidades de publicar. En este sentido, existen periódicos que solo publican el mínimo requerido por el respectivo índice donde están registrados (alrededor de seis), pero otras optan por incorporar hasta veinticinco o más artículos por número.

5. Factor de impacto (FI): El FI mide la frecuencia con la que una publicación ha sido citada en un año. Este indicador permite comparar unas con otras y evaluar su rango dentro del mismo campo científico. Cuanto mayor sea el FI, más exigentes serán las evaluaciones para la aceptación de un artículo y, por lo tanto, más difícil será lograr la publicación. Éste calcula dividiendo el número de citas del año correspondiente al JCR (Journal Citation Report) (numerador), entre el número total de artículos citables publicados en los dos años anteriores (denominador) [9].

6. Línea editorial (LE): Constituye el perfil, las orientaciones, los temas o el campo disciplinar que se priorizan. Lógicamente si el artículo enviado no se ajusta a la LE es muy difícil que sea aceptado. 
7. Tipo de arbitraje: La gran mayoría de las revistas utilizan el sistema de revisión "doble ciego", o sea, los evaluadores y los autores no se conocen (se garantiza el anonimato). En este proceso, la generalidad emplea a dos evaluadores, mientras otras solicitan tres e incluso cuatro "evaluadores ciegos".

Ello implica que para la aceptación de un trabajo solo incide su valía y aportes. Evidentemente, dependiendo de la cantidad de revisores, o "evaluadores ciegos", aumenta o disminuye la dificultad para materializar con cierta velocidad el proceso de las publicaciones.

8. Especificidad o especialidad de la revista: Si el artículo redactado disciplinariamente es de alta calidad, quizás convenga intentar publicarlo en una revista especializada.

Si bien las revistas con una temática más amplia pueden no ser tan exigentes (desde el punto de vista de la línea disciplinaria), igualmente reciben gran cantidad de trabajos, lo que disminuye las posibilidades de lograr publicar.

9. Lugar de la revista: Considerar si la revista a la que se envió el estudio es de otro o del mismo país de los autores, o si pertenece a la institución donde estos se desempeñan o no, ello por el tema de la endogamia académica. Normalmente, si la revista es de otro país o institución podría influir positivamente en la oportunidad de publicar.

10. Soporte que entrega la revista: Existen muchas revistas académicas que apoyan a los autores no solo en la edición final del artículo, sino también en la traducción del resumen y su revisión y corrección (generalmente cuando tiene ciertos detalles que se alejan de las pautas o instrucciones a los autores). Habitualmente en estas revistas, la publicación de un artículo tiende a demorarse menos tiempo.

11. Tipos de artículos: Es conveniente conocer la naturaleza de los trabajos qué demanda una revista en la que se intenta publicar; cumplir con este requerimiento aumenta la posibilidad de aceptación. Unas reciben solo artículos de investigación; otras abren su espectro a revisiones, ensayos (escritos por académicos e investigadores de reconocida trayectoria), estudios de casos, notas técnicas, reseñas, entre otros.

\section{Recomendaciones adicionales}

1. Solicitar opiniones de expertos: También es útil considerar la posibilidad de que colegas y profesionales del área puedan leer y dar su opinión acerca del artículo, lo cual aumenta las posibilidades de lograr la publicación y, eventualmente, de generar retroalimentación con otros investigadores, lo que puede enriquecer o refutar nuestro aporte. Este posible intercambio mejoraría la calidad del conocimiento generado y los beneficios que puede obtener la sociedad a partir de ellos.

2. Redacción y gramática: Poner especial cuidado en escribir de manera clara y directa y respetar las normas gramaticales del idioma. Sin omitir términos técnicos y/o científicos, se debe evitar los tecnicismos y redactar de forma tal que el texto pueda ser comprendido, tanto por expertos en el tema, como también por estudiantes, docentes o cualquier otro posible lector que esté buscando bibliografía sobre la materia. Lograr una redacción atractiva, que sin crear falsas expectativas, motive a consultar el artículo.

3. Respetar rigurosamente las normas de publicación: Algo a lo que muchas veces no se le da mayor valor y que nunca puede ser subestimado son los criterios formales predeterminados por cada Comité Editorial.

Al respecto, Heredia [10] destaca que una meticulosa preparación del artículo, que se ciña a las normas preestablecidas por la revista y al rigor científico necesario, facilita la tarea de quienes evalúan el artículo. Se puede mencionar entre estos a los editores y supervisores, que son científicos que contribuyen a la ciencia también desde este rol, y a los revisores o árbitros, que son investigadores pares de los autores. Los editores, cara pública de la revista, tienen la importante función de decidir qué va a ser publicado y establecer las políticas editoriales en conjunto con el comité editor y el responsable de la editorial. A su vez, también coordinan el proceso de revisión de pares.

4. Asociarse a investigadores consolidados: Cuando se decide generar conocimiento y divulgar esos saberes es trascendente unirse a equipos de investigación afianzados, liderados por personas con experiencia y alto nivel y volumen de publicaciones. Si se trabaja con investigadores versados (no noveles), con su prestigio, su aporte y recomendaciones se conseguirá 
armar un artículo ajustado a los requerimientos de publicación y avalado por nombres reconocidos.

Mario Bunge [11] registra que Robert K. Merton, el padre de la moderna sociología de la ciencia, bautizó con el nombre de "efecto San Mateo" al hecho de que los investigadores científicos eminentes cosechan aplausos mucho más nutridos que otros investigadores menos conocidos, por contribuciones equivalentes. Por ejemplo, si un autor famoso F colabora con un desconocido $\mathrm{D}$, en una labor hecha casi exclusivamente por $\mathrm{D}$, la gente tiende a atribuirle todo el mérito a F. Por cierto, el maestro le hace un favor al aprendiz al firmar juntamente un trabajo: lo lanza (como le decía el Quijote de la Mancha a Sancho: "quien a buen árbol se arrima, buena sombra le cobija"). En definitiva, el nuevo investigador se beneficiará de la reputación y práctica del "investigador senior", hasta que se transforme en referente, y pueda él también "servir de paraguas", para los nuevos investigadores que estén emergiendo.

5. Incorporarse a redes académicas: La generación de redes entre investigadores e instituciones (universidades, entidades no lucrativas, empresas u organizaciones del sector público) es muy acertado. En el caso de la articulación público-privada, es beneficioso que la contraparte cuente con personal formado para que pueda actuar con las competencias pertinentes en emprendimientos de investigación conjunta:

“...es necesario gestionar con eficiencia el conocimiento, pues es solo a partir del desarrollo de esta capacidad que 'las redes científicas, o redes del conocimiento, en las que participan tanto generadores del conocimiento (investigadores) como usuarios del mismo', se pueden constituir en 'la espina dorsal de las nuevas sociedades"” [12], [6].

En este sentido, la conformación de comunidades científicas (programas, núcleos, agrupaciones, centros y/o institutos de investigación) resulta indispensable para facilitar la apropiación social del conocimiento. A su vez, la articulación de las universidades con los gobiernos y con empresas que tengan una genuina responsabilidad social es fundamental para sustentar las políticas públicas a implementar.

Por otra parte, es evidente que el desarrollo de las nuevas tecnologías de la información y de la comunicación (TICs) han eliminado las barreras témporo/espaciales viabilizando el contacto en tiempo real y permitiendo compartir cualquier formato de archivo, de texto, audio y video en cualquier momento. Estas herramientas facilitan el trabajo en equipo con pares de cualquier lugar del planeta y poner al alcance de millones de personas los resultados de las investigaciones.

Algunos ejemplos de redes mundiales de información, pueden visualizarse en la Tabla 1. Cabe mencionar que muchas de las organizaciones de dicha tabla promueven listas abiertas de discusión.

6. Considerar costos involucrados: En el universo de las revistas académicas, existen un amplio número que no exigen ningún pago por publicar en ellas, existiendo incluso algunas que retribuyen a los autores que logran que su artículo sea aceptado

Tabla 1. Algunas redes internacionales a considerar.

\begin{tabular}{|c|c|c|}
\hline Nombre de organización & Sigla & Dirección web \\
\hline Organización para la Cooperación y Desarrollo Económicos & OCDE & www.oecd.org \\
\hline Centro Latinoamericano de Administración para el Desarrollo & CLAD & www.clad.org \\
\hline Consejo Latinoamericano de Escuela de Administración & CLADEA & www.cladea.org \\
\hline Organización de las Naciones Unidas para la Alimentación y la Agricultura & FAO & www.fao.org \\
\hline Fondo de las Naciones Unidas para la Infancia & UNICEF & www.unicef.org \\
\hline $\begin{array}{l}\text { Organización de las Naciones Unidas para la Educación, la Ciencia y la } \\
\text { Cultura. Comisión Económica para América Latina y el Caribe }\end{array}$ & $\begin{array}{l}\text { UNESCO } \\
\text { CEPAL }\end{array}$ & $\begin{array}{l}\text { www.unesco.org } \\
\text { www.cepal.org/es }\end{array}$ \\
\hline Programa de las Naciones Unidas para el Desarrollo & PNUD & www.undp.org \\
\hline $\begin{array}{l}\text { Organización Internacional del Trabajo } \\
\text { Banco Mundial }\end{array}$ & $\begin{array}{l}\text { OIT } \\
\text { Banco }\end{array}$ & $\begin{array}{l}\text { www.ilo.org } \\
\text { www.worldbank.org }\end{array}$ \\
\hline
\end{tabular}


para la correspondiente publicación; sin embargo, también coexisten otras que solicitan importes por maquetación, sometimiento a evaluación, número de páginas publicadas que tiene el artículo, entre otros puntos.

A modo de síntesis, en la Figura 3 se presentan de manera esquemática los diversos elementos a tomar en cuenta al momento de escribir y publicar un artículo académico.

\section{Estructura del artículo: guía a tener en cuenta} La organización de la información varía de acuerdo al tipo de publicación. Las revistas especializadas no solamente publican artículos de investigación científica, favorecen además el diálogo entre pares por medio de otras formas textuales: reviews (artículos de revisión), cartas al editor, reseñas bibliográficas, ensayos, traducciones, entre otros.

En términos generales, los artículos de investigación científica se estructuran del siguiente modo: el título, el resumen, las palabras clave y la introducción, los fundamentos teóricos, la metodología utilizada, los resultados y la discusión, y, por último, las conclusiones y el listado de referencias bibliográficas, además de las secciones de forma que incluyen nombres de los autores, datos filiatorios y agradecimientos (ver Figura 4).

Es primordial prestar atención a algunas características anteriores por su vital incidencia en la calidad de una publicación.

\section{Título}

Se debe considerar que esta parte es como una especie de "tarjeta de presentación", brinda la primera impresión, posibilita que el lector pueda identificar el tema con facilidad. Debe ser atractivo y conciso, con una indicación esclarecedora sobre el contenido. No es aconsejable el uso de terminología altamente técnica, abreviaturas y frases en otro idioma (verbi gratia: inglés o francés, si el artículo está escrito en español), salvo que no exista una traducción adecuada. El título debe representar muy bien el objetivo general, lo que es una clara señal que permite mostrar desde el comienzo la coherencia interna del artículo. Dependiendo de la revista, podría tener una extensión de doce a veinte palabras.

\section{Autor(es)}

En esta sección la mayoría de las revistas solicitan una serie de datos como: nombre completo,

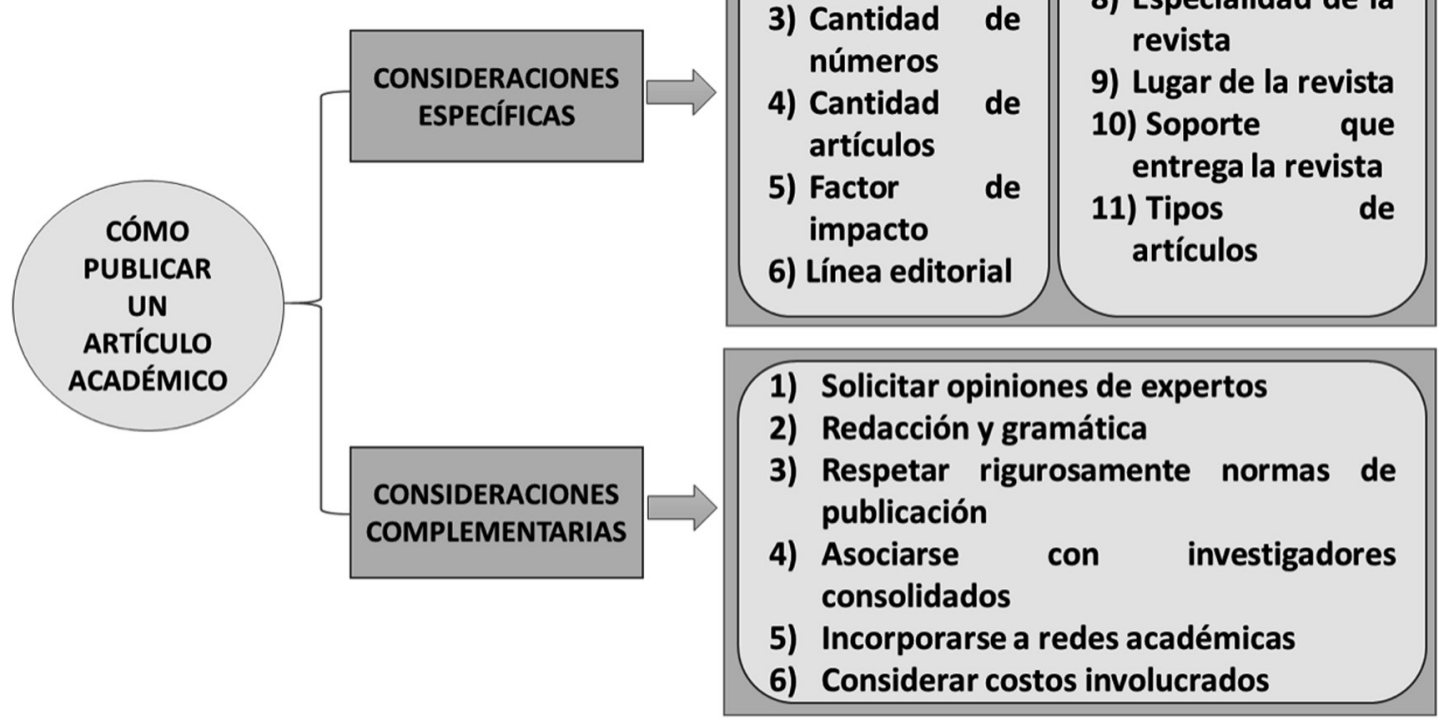

Figura 3. Consideraciones para la publicación de un artículo académico.

Fuente: Diseño propio, basado en Ganga, Paredes y Pedraja [8]. 

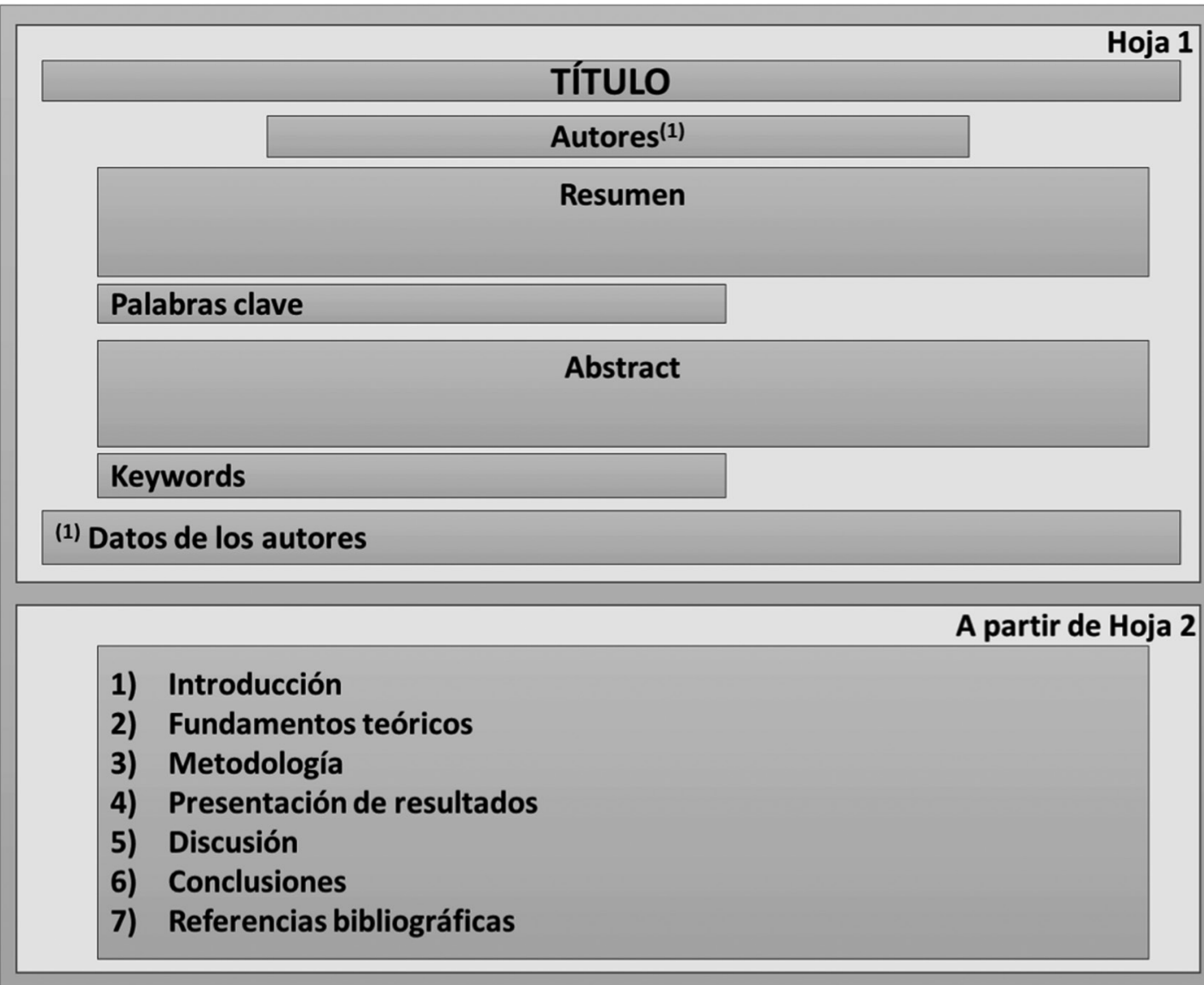

Figura 4. Estructura de un artículo.

Fuente: Diseño propio, basado en artículos publicados en revistas académicas.

dependencia organizacional, título(s) y grado(s) académicos; además de dirección y correo electrónico.

\section{Resumen}

En la mayoría de las bases de datos este se publica separadamente como una especie de anunciación del trabajo. Depende de la claridad de su redacción que el lector pueda identificar rápidamente de qué se trata el estudio y valorar si es pertinente a su búsqueda.

Se recomienda comenzar con unas dos líneas de contexto, aproximadamente 40 a 80 palabras (a lo más 120 palabras, dependiendo del tamaño total del resumen), y luego, referirse sintéticamente al objetivo de la investigación en más o menos unas 30 a 60 palabras. De inmediato describir brevemente la metodología utilizada para conseguir el propósito central (alrededor de 30 a 50 palabras), y los principales resultados alcanzados y/o la conclusión más relevante que se pueda destacar (alrededor de 20 a 40 palabras).

La extensión completa del resumen depende de los requerimientos de la revista a la que se envíe el artículo, debe contar con 150 palabras como promedio, sin embargo, hay algunas que solo aceptan 90 y otras hasta 250 .

Habitualmente se pide en español e inglés o viceversa, aunque últimamente muchas lo solicitan en tres y cuatro lenguas.

\section{Palabras clave}

Se ubican a continuación del resumen, y tienen un significado cardinal ya que son utilizadas para indexar el artículo en las bases de datos a las que 
están suscritas las revistas. Pueden constituir términos únicos o grupos de palabras.

Se debe evitar el uso de abreviaturas, fórmulas, siglas o acrónimos, así como repetir las palabras del título, con el objetivo de incrementar las posibilidades del buscador.

Las revistas piden entre tres y siete palabras clave por lo general, y al igual que el resumen, estas deben traducirse del español al inglés o viceversa, aunque muchas publicaciones solicitan tres y cuatro idiomas.

\section{Introducción}

Se podría decir que la introducción es una especie de resumen extendido y complementado. Por lo anterior, se recomienda comenzar con un par de párrafos que contextualicen el trabajo realizado (50 a 100 palabras aproximadamente); acto seguido, se puede continuar con dos o tres párrafos relacionados con los fundamentos teóricos que sustentan el trabajo (100 palabras a 200 aproximadamente), lo que no es obligatorio para el caso de las ciencias experimentales; para luego dar paso a la presentación del objetivo más relevante de la investigación (40 palabras a 90 aproximadamente); finalizando con una presentación de la metodología usada para lograr el propósito cardinal planteado (aproximadamente 30 a 90 palabras). En algunos casos se recomienda incorporar un párrafo adicional que adelante cada una de las partes que se encontrarán al leer el artículo académico (aproximadamente entre 50 a 90 palabras); sin embargo, esto no es requerido en aquellas disciplinas que emplean metodologías experimentales.

\section{Fundamentos teóricos}

En algunas disciplinas se debe considerar una revisión bibliográfica que permita situar conceptualmente al lector y que instituya las bases teóricas sobre las que se hará el análisis. Esta sección se puede ordenar cronológicamente o por temas, con el fin de permitir un mejor entendimiento del problema bajo estudio [13].

No obstante lo anterior, cabe destacar la importancia de realizar una revisión de investigaciones similares y de sus resultados, ya que es preciso contar con una noción general de lo que se ha hecho en esa área del conocimiento.

\section{Metodología}

Describe los procedimientos esenciales y herramientas utilizados para el logro de los objetivos planteados.

Básicamente, se expone el diseño general de la pesquisa que se ha llevado a cabo; corresponde a un desglose de los pasos dados para analizar la información recopilada por medio de la investigación.

Describir la metodología es elemental, esta debe ser redactada de manera precisa y suficientemente detallada como para que cualquier otro investigador pueda conocer cómo se llevó a cabo el estudio.

Si bien su estructura interna puede variar dependiendo de la naturaleza de la investigación, prevalecen los siguientes pasos: variables, dimensiones y medidas; unidad y nivel de análisis; población y tamaño de la muestra; instrumento para la recolección de datos; selección de los casos; método de análisis; entre otros [14].

\section{Presentación de resultados}

Muestran los principales hallazgos obtenidos en el proceso de investigación los que son analizados desde una perspectiva de diálogo con otros autores especialistas en la temática.

No es necesario reportar cada detalle de los resultados, por lo que el foco debe mantenerse en aquellos que agregan valor a las hipótesis o teorías planteadas por la investigación misma [15]. Adicionalmente, para una mejor comprensión de los resultados se recomienda hacer uso de tablas, gráficos, figuras $\mathrm{u}$ otras herramientas que permitan exponer los hallazgos de una forma más simplificada.

Lopez [16], [12], recomienda que esta parte contenga lo siguiente: referencia bien fundamentada al propósito del estudio, a la hipótesis central; revisión de los resultados en orden de importancia, resaltando si estos sustentan en forma adecuada la hipótesis principal del trabajo y contestan las preguntas de investigación y alcanzan los objetivos propuestos; explicación de los resultados soportados en forma suficiente por la sólida y actualizada bibliografía manejada; establecimiento de las limitaciones del estudio, las cuales evitan la aplicación generalizada de los resultados; implicaciones del estudio, tanto desde la perspectiva del contexto del espacio, de 
tipo referencial, como desde la perspectiva teórica $\mathrm{y}$; recomendaciones para futuras investigaciones (esta última, en algunas revistas lo solicitan en la parte de las conclusiones.

\section{Conclusiones}

En esta sección, se exponen los aportes más relevantes del campo disciplinar, las posibles aplicaciones, las líneas de investigación futuras y las principales limitaciones (debiera tener entre 350 a 450 palabras aproximadamente).

Las conclusiones deben ser estimadas como la fase final de las diversas etapas que comprendió el proceso de estructuración del artículo. Es muy provechoso, antes de redactarlas, volver a leer-las veces que sea necesario-concentrada, detallada y reflexivamente todo el texto que se pretende publicar.

Habitualmente debieran dar a conocer, de la manera más clara y objetiva posibles, tanto las fortalezas o potencialidades como las vulnerabilidades del trabajo, se podrían mencionar brevemente los aspectos metodológicos utilizados, y hacer referencia a su influencia en el contenido del estudio; para luego enunciar las futuras líneas de investigación que conseguiría generar el artículo.

Las conclusiones de ninguna manera pueden convertirse en una repetición de los resultados o hallazgos encontrados. Estas deben exponer la contribución final del estudio realizado y las posibles futuras líneas de investigación [17].

\section{Agradecimientos}

Como la producción científica normalmente se realiza en equipos de trabajo, o con la colaboración de personal especializado y/o de determinadas organizaciones, la mayoría de las revistas permiten que los autores puedan reconocer formalmente estos aportes, en una extensión que fluctúa entre 20 a 40 palabras aproximadamente.

\section{Referencias bibliográficas}

La bibliografía o referencias constituyen la fuente de alimentación del estudio, su sustento teórico. Esta debe estar actualizada y ajustarse a lo que realmente se consultó.

De acuerdo a Rivera-Camino [18], aunque los títulos: Bibliografía, Referencias y Literatura
Citada, se emplean frecuentemente como sinónimos, el primero debe usarse cuando se presenta una recopilación completa de la literatura, el segundo cuando se presenta una selección de artículos y el tercero cuando todos los artículos citados en el texto aparecen en la lista de referencias y viceversa.

A su vez, American Psychological Association (APA) [19], indica que en las referencias se incluyen solo aquellas anotaciones que se encuentran dentro del cuerpo del artículo científico en donde se específica el autor de la idea, cita o párrafo que se está utilizando y, a su vez, en la bibliografía se recopilan todas las fuentes que fueron necesarias para la realización del trabajo escrito.

Más allá de esta adecuada precisión es apropiado considerar que no existen pautas o patrones consensuados universalmente para proceder a su confección, por lo mismo, se deben seguir exhaustivamente las "pautas o instrucciones para los autores", que tiene la revista donde se desea publicar.

En el momento de la búsqueda y la recopilación de la información es muy conveniente anotar los datos de cada una de las fuentes revisadas de las que se ha utilizado y/o extraído información:

Autor(es), ya sea persona o institución.

Título de la publicación y Subtítulo (cuando el subtítulo es relevante).

Número de edición (excepto si es la primera).

Lugar de publicación.

Editorial y año de publicación.

Páginas (si se trata de obras de más de un volumen, se debe anotar el número de estos sin indicar las páginas). Nota de serie.

Tipo de soporte.

Fecha de consulta (requerido para documento en línea).

Conservar estos datos permitirá tener identificada y ubicada la fuente de la que estamos obteniendo información y que puede ser parte de la bibliografía.

Para su presentación y orden las revistas comúnmente utilizan normas como las de: American Society of Civil Engineers (ASCE), de Institute of Electrical and Electronics Engineers (IEEE), American Society of Mechanical Engineers (ASME), American Chemical Society (ACS), American Psychological Association (APA), por mencionar algunas de las más usadas. 


\section{CONCLUSIONES}

El ciclo de una investigación culmina y toma su mayor sentido cuando esta es publicada, y los resultados de una meticulosa, paciente, y muchas veces larga faena, de uno o más investigadores logran salir a la luz, estar al servicio de la comunidad científica y de toda la sociedad.

La generación de nuevo conocimiento va de la mano con el incremento de las cifras y la calidad de las publicaciones científicas que se realizan. En este sentido, las instituciones educativas juegan un rol fundamental al promover la formación de investigadores, motivar y capacitar a sus docentes para realizar esta tarea e incentivar -en el seno de sus organizaciones- la consolidación de una cultura que propenda a aumentar las publicaciones científicas de excelencia. Este cambio involucra también a otros actores sociales de relevancia como los gobiernos y el sector privado, dado que la generación de conocimiento es de importancia estratégica para el desarrollo de las naciones.

El uso de las tecnologías de la información ofrece las herramientas para aunar esfuerzos por medio de la generación de redes académicas que faciliten la labor. Posibilitan además la generación de vasos comunicantes que conectan a investigadores de diferentes lugares del mundo para compartir experiencias.

Por otra parte, la implementación de políticas universitarias y gubernamentales que tiendan a la consolidación de grupos de investigación dispuestos y preparados para llevar adelante líneas encaminadas a la solución de los problemas regionales y nacionales resulta de absoluta preeminencia para el progreso de las sociedades modernas.

Tomando como referencia lo expuesto anteriormente se pretende que las recomendaciones ofrecidas para la estructuración de un artículo científico puedan servir de guía a quienes desean incursionar en la publicación de sus investigaciones.

En este orden de cosas se espera que este trabajo contribuya y despeje el camino de aquellos que intentan publicar en revistas indexadas.

Junto con reconocer los aportes potenciales de este trabajo, se considera de utilidad indicar que una serie de cuestiones podrían mejorarse en futuros estudios; verbi gratia, aplicar instrumentos de recolección de información (encuestas, entrevistas, etc.) que permitan conocer la percepción de investigadores consolidados, de docentes que nunca hayan publicado y de los propios estudiantes para estar al tanto del enfoque de todos desde sus respectivas posiciones.

Puede resultar provechoso, además, contar con un catastro de revistas que se pudieran recomendar, o crear una especie de base o espacio de conexión que facilite la publicación a nuevos colegas, especialmente de Latinoamérica.

\section{AGRADECIMIENTOS}

Los autores agradecen al Centro de Estudios de la Universidad de Tarapacá (CEUTA) de Arica-Chile y los aportes de la Bibliotecóloga Lilia Sánchez de la Universidad de Los Lagos.

\section{REFERENCIAS}

[1] E. Ander-Egg. "Técnicas de Investigación Social". Lumen. 24a edición. Buenos Aires, Argentina, pp. 425. 1995. ISBN: 9507245138.

[2] Real Academia Española - RAE. URL: http:// www.rae.es/. Fecha de consulta: Junio de 2015.

[3] G. Gómez de Silva. "Breve Diccionario Etimológico de la Lengua Española”. S.L. Fondo de Cultura Económica de España. $2^{\mathrm{a}}$ edición, $9^{\circ}$ reimpresión. México, pp. 736. 2013. ISBN: 9789681655433.

[4] C. Gutiérrez y P. Mayta. "Publicación desde el Pre Grado en Latinoamérica: Importancia, Limitaciones y Alternativas de Solución". CIMEL. Vol. 8 No 1, pp. 54-60. 2003.

[5] R. Day. "Como escribir y publicar trabajos científicos. Organización Panamericana de la Salud”. Publicación Científica. № 526. Estados Unidos de Norteamérica. 1990.

[6] A. Romero. "Las redes de información y su importancia para la investigación científica". Revista Venezolana de Gerencia, Universidad del Zulia. Vol. 7 No 19, pp. 425-441. 2002. URL: http://www.redalyc.org/articulo. oa?id=29001906. Fecha de consulta: Agosto de 2015.

[7] K. Gibrán. "La voz del maestro". Longseller S.A. $1^{\text {a }}$ edición. Buenos Aires, Argentina. 2004. 
[8] F. Ganga, L. Paredes y L. PedrajaRejas. "Importancia de las publicaciones académicas: Algunos problemas y recomendaciones a tener en cuenta". Idesia. Vol. $33 \mathrm{~N}^{\circ}$ 4, pp. 111-119. 2015.

[9] Thomson Reuters. "Factor de impacto de una revista". Julio de 2016. URL: http://wokinfo. com/media/pdf/Impact_Factor_2016_ release_SPANISH.pdf

[10] A. Heredia. "¿Por qué y cómo publicar artículos científicos en revistas internacionales?". Seminario para Autores - Elsevier Latinoamérica. Santiago, Chile. Agosto de 2010.

[11] M. Bunge. "El efecto San Mateo". Revista Polis [En línea]. $N^{\circ}$ 2. 2002. URL: https:// polis.revues.org/8033. Fecha de consulta: 1 de abril de 2016. DOI: 10.4000/polis.8033.

[12] F. Chaparro. "Haciendo de Colombia una Sociedad del Conocimiento. Conocimiento, Innovación y Construcción de Sociedad: Una Agenda para la Colombia del Siglo XXI". Colciencias, TM Editores. Santafé de Bogotá, Colombia, pp. 120. 1998. ISBN: 9586018237.

[13] University of Southern California (USC) Writing Center. "Organizing Your Social Sciences Research Paper”. 2016. URL: http:// libguides.usc.edu/writingguide. Fecha de consulta: 11 de abril de 2016.

[14] I. Corrales-Reyes, M. Rodríguez-García, J. Reyes-Pérez y M. García-Raga. “Cómo redactar un artículo científico?". Revista 16 de Abril. Vol. $54 \mathrm{~N}^{\circ}$ 258, pp. 4-17. 2015.

[15] L. Schwabe, G. López-Bendito and C. Ribeiro. "Getting published: how to write a successful neuroscience paper". European Journal of Neuroscience. Marzo de 2016. URL: http:// onlinelibrary.wiley.com/doi/10.1111/ejn.13215/ full. Fecha de consulta: 11 de Abril de 2016. DOI: 10.1111/ejn.13215.

[16] S. López. "El proceso de escritura y publicación de un artículo científico". Revista Electrónica Educare. Enero-abril. Vol. 17 $\mathrm{N}^{\mathrm{o}}$ 1, pp. 5-27. 2013.

[17] Elsevier. "How to write a world class methodology paper: Tips, traps and travesties". URL: http://www.utsa.edu/ 1rsg/teaching/geo6011/howtowritepaper. pdf. Fecha de consulta: 2 de abril 2016.

[18] J. Rivera-Camino. "Cómo escribir y publicar una tesis doctoral". ESIC Editorial. $2^{a}$ edición, pp. 268. Madrid, España. 2014.

[19] Normas APA-Ed. 6. URL: http://normasapa. net/normas-apa-2016/ Fecha de consulta: 20 de agosto 2016. 